\title{
El recuerdo de hechos traumáticos: exactitud, tipos y características
}

\section{Memories for traumatic events: accuracy, types and characteristics}

\section{Resumen}

El estudio de las memorias traumáticas ha generado un importante debate debido a su implicación en la comprensión del funcionamiento de la memoria y la importancia de los recuerdos traumáticos en entornos forenses. Se pretende con esta revisión entender las causas que nos pueden ayudar a explicar el debate en torno a las memorias traumáticas, analizando la definición de trauma y los factores que pueden condicionar su recuerdo. Por último, y debido a su importancia en contextos forenses, se realizó un análisis en torno a la exactitud de las memorias traumáticas. La explicación en torno a cómo recordamos un hecho traumático está en función de la concepción del término "trauma". Si el trauma se entiende por los efectos psicológicos que provoca, nos encontraremos con estudios que abogan por las memorias diferenciales. Si definimos el trauma por las características del agente que lo provoca, entonces nos encontraremos con estudios que apuntan a las teorías de las memorias equiparables. Independientemente de dónde se ponga el foco, habrá que considerar los factores que influyen en el recuerdo, fundamentalmente el estrés, la intensidad de la emoción asociada o el grado de implicación. Su efecto condicionará la capacidad para desplegar las estrategias de afrontamiento necesarias y son importantes para entender las diferencias en la exactitud de los recuerdos y en la accesibilidad a las memorias traumáticas.

Palabras clave: Memoria autobiográfica. Trauma. Emoción. Trastorno de estrés post-traumático.

\section{Abstract}

The study of memory for trauma has generated considerable controversy. Its importance stems from its involvement in the understanding of memory processes, and for its importance in forensic contexts. The aim of this study was to analyze the factors that help explain the controversy surrounding memories for trauma. To do this the definition of trauma and the factors that can determine the memory were analyzed. Also, the accuracy of memories for trauma was analyzed. The explanation of memory for trauma is depending on the concept of trauma. If trauma is defined by its psychological effects, then studies support that memories for trauma are different from other autobiographical memories. If trauma is defined by the characteristics of its cause, then studies support that memory for trauma are different from other memories. Regardless of focus, factors that influence the recall should be considered. Stress level, intensity of emotion, and degree of involvement are the most important. The effect of these factors affects the ability to deploy strategies to face the trauma, and it is important to understand the differences in the accuracy and accessibility of memories.

Key words: Autobiographical memory. Trauma. Emotion. Post-Traumatic Stress Disorder.

El presente trabajo forma parte del proyecto de investigación "Entrevista, intervención y criterios de credibilidad en abusos de carácter sexual en personas con discapacidad intelectual" que se lleva a cabo en la Fundación Carmen Pardo-Valcarce, con la colaboración de la Fundación MAPFRE, el Ministerio de Interior y la Universidad Complutense de Madrid.

\section{AL. Manzanero ${ }^{1}$ M. Recio ${ }^{2}$}

${ }^{1}$ Universidad Complutense de Madrid (España). ${ }^{2}$ Fundación Carmen Pardo-Valcarce (España).

Correspondencia: Antonio L. Manzanero Departamento de Psicología Básica I Facultad de Psicología Universidad Complutense de Madrid 28223 Madrid

E-mail:

antonio.manzanero

@psi.ucm.es

Fecha de recepción: 20.FEB.2012

Fecha de aceptación: 16.MAR.2012 


\section{Introducción}

Se definen las memorias traumáticas como recuerdos sobre hechos con una valencia negativa y alto impacto emocional. El impacto que los hechos traumáticos tienen sobre las personas depende de diferentes factores, existiendo importantes diferencias individuales, que determinarán la experiencia fenomenológica asociada al recuerdo del suceso vivido. En algunos casos, estos sucesos pueden dar lugar a un trastorno de estrés post-traumático, que se caracteriza por la tendencia en las personas que la sufren a la re-experimentación (vivencias intrusivas como recuerdos del acontecimiento y sueños sobre el mismo o sensación de estar ocurriendo de nuevo), la evitación (esfuerzos por evitar pensamientos, sentimientos, recuerdos, lugares o personas relacionados con el suceso) y la hiperactivación (dificultad para dormir, irritabilidad o explosiones de rabia).

A largo plazo, los efectos dependerán de la intensidad del trauma y de la vulnerabilidad del sujeto. Así, no afectará de la misma manera un trauma derivado de un accidente de tráfico que un trauma derivado de una experiencia de abuso sexual continuado en la infancia. De igual modo, se han propuesto tres factores claves en la respuesta a una experiencia traumática:

a) las experiencias de apego negligentes $u$ otros traumas en la infancia,

b) las estrategias de afrontamiento, $y$

c) el apoyo social a la víctima del trauma ${ }^{1-3}$.

\section{La especificidad de las memorias traumáticas}

Una revisión reciente ${ }^{4}$ propone que la controversia en relación a las memorias traumáticas puede resumirse en varios puntos fundamentales: a) si estas memorias son diferentes de otros tipos de memorias autobiográficas, b) si las memorias traumáticas son más o menos exactas que las memorias de hechos no traumáticos, y c) si estas memorias pueden olvidarse completamente y recordarse mucho tiempo después.

Podemos distinguir diferentes propuestas en torno a las características diferenciales de las memorias traumáticas: a) las que señalan que las memorias traumáticas serían diferentes a otras memorias autobiográficas, y b) las que afirman que ambos tipos de memorias son similares. A continuación explicaremos brevemente ambas propuestas así como las principales investigaciones publicadas que las sustentan.

\section{Las memorias sobre hechos traumáticos: un tipo de memoria especial}

Algunos estudios muestran que las memorias traumáticas tienen características diferentes a las memorias sobre otros hechos autobiográficos. En este marco, se han señalado dos propuestas diferentes: a) las memorias de hechos traumáticas son mejores que las memorias neutras y $b$ ) las memorias de hechos traumáticas son peores que las memorias neutras.

Con respecto a la primera propuesta, diferentes investigaciones han puesto de manifiesto la aparente superioridad de las memorias sobre hechos traumáticos ${ }^{5-9}$. Según estos estudios, sentimos que somos capaces de recordar estos sucesos como si acabaran de ocurrir, aparentando ser inmunes al deterioro producido por el paso del tiempo. Este tipo de memorias ha sido denominado memorias vívidas (flashbulb memories). Un hecho de este tipo es, por ejemplo, el atentado ocurrido en Nueva York el 11 de septiembre de 2001, cuyo impacto emocional no deja lugar a duda. Cuando recordamos aquel día y lo que nosotros hacíamos antes, durante y después del atentado es muy probable que tengamos la sensación de que aquello se nos ha quedado profundamente grabado, que lo recordamos de forma muy vívida y con todo lujo de detalles.

En esta dirección, algunas investigaciones ${ }^{10}$ han mostrado que los hechos traumáticos se recuerdan mejor tres meses después que los que no lo son. Peace, Porter y Brinke ${ }^{11}$, tras comparar memorias reales sobre agresiones sexuales, traumas y hechos no traumáticos, encontraron que las primeras eran más vívidas, detalladas y sensoriales que las segundas. Así pues, los acontecimientos traumáticos se recordarían mejor, serían más consistentes con el paso del tiempo, y se podrían caracterizar por una memoria clara y exacta para los detalles centrales del suceso ${ }^{12,13}$, aunque con poca exactitud para los detalles irrelevantes, de forma más acentuada a lo que igualmente ocurre con los recuerdos de otros hechos autobiográficos.

En contra de esta propuesta, algunos autores afirman que este tipo de memorias serían peores que las memorias sobre otro tipo de hechos autobiográficos, ya que pueden presentarse fragmentadas ${ }^{14-18}$ y ser difíciles de expresar de forma narrativa, aunque pueden estar asociadas a sensaciones intensas (olorosas, auditivas, táctiles...), y ser muy visuales ${ }^{19-21}$. Incluso se ha propuesto que este tipo de memorias podría estar asociado a episodios de amnesia, siendo más susceptible de aparecer ante emociones extremas, 
como las que pueden despertar los delitos pasionales ${ }^{22}$, y suelen estar asociadas a casos de amnesias transitorias de origen orgánico e intoxicaciones por alcohol $^{23}$.

Las propuestas sobre memorias fragmentadas de hechos traumáticos están sustentadas, además, en los estudios experimentales y clínicos sobre el trastorno de estrés postraumático ${ }^{24,25}$, cuyos síntomas relacionados con el recuerdo (el olvido de algunos aspectos del suceso, y el recuerdo vívido y persistente de otros momentos del mismo en forma de "flashbacks") ilustran este tipo de memoria traumática fragmentada.

\section{Datos para una propuesta sobre la inespecifi- cidad de las memorias traumáticas}

Algunos autores ${ }^{26}$ afirman que pese a existir diferencias en algunos aspectos entre las memorias autobiográficas traumáticas y neutras, no implican diferencias sustanciales en sus características ni en su deterioro. Varios estudios recientes ${ }^{27-29}$ parecen confirmar estas afirmaciones con poblaciones distintas. En el primer estudio se compararon los recuerdos de hechos traumáticos (fallecimientos, agresiones, separaciones, accidentes, atentados y otros) con recuerdos de hechos felices (nacimientos, actividades de ocio, bodas, trabajo, reencuentros y otros), mediante el Cuestionario sobre Características Fenomenológicas de Recuerdos Autobiográficos (CCFRA), diseñado al efecto. Este estudio se realizó con población española y se consideraron 120 recuerdos. En general, los resultados mostraron que los recuerdos de hechos traumáticos en comparación con hechos felices se caracterizaban por contener menos información sensorial, ser más complejos, más difíciles de fechar, con sentimientos asociados más intensos, un mejor recuerdo de pensamientos asociados en el momento de su ocurrencia, más difíciles de expresar verbalmente y con más pensamientos recurrentes sobre lo ocurrido. Por el contrario, no se encontraron diferencias significativas sobre localización espacial del suceso, vividez, definición, accesibilidad, fragmentación, perspectiva de recuperación, dudas sobre su ocurrencia, ni tendencia a hablar sobre lo ocurrido.

¿Por qué hay conclusiones tan dispares? Una de las razones que explica esta disparidad es que la misma concepción de lo que se está estudiando varía de unos estudios a otros, es decir, si el trauma se define más por sus efectos psicológicos en las víctimas, nos encontramos con las propuestas de memorias diferenciales. Pero si el foco de la defini- ción del trauma recae sobre las características intrínsecas de los agentes que los provocan, entonces aparecerían más las memorias equiparables. Lo cierto es que desde su origen el trauma se ha definido no tanto por el hecho en sí como por los efectos físicos o psicológicos que ha tenido en las víctimas. El trauma desborda la capacidad del individuo de tolerar y procesar las emociones que genera y desorganiza las estrategias de una persona para manejarse en la vida. Un atentado, un accidente de coche o un maremoto puede ser o no traumático, dependiendo de los efectos psicológicos que haya desencadenado. Pero también se puede aceptar que los tres son traumáticos por el hecho de constituirse como una amenaza a la integridad. Independientemente de dónde situemos el foco, al analizar la literatura al respecto, lo primero que deberemos considerar es qué concepción de trauma les acompaña, porque sólo así podremos empezar a entender las disparidades en el estudio de las memorias traumáticas.

En cualquier caso, las opiniones tan diversas en torno a las características de las memorias traumáticas con respecto a otras memorias autobiográficas nos llevan a plantearnos la necesidad de ser prudentes a la hora de generalizar conclusiones acerca de la manera de recordar un hecho traumático. Parece razonable concluir, a la luz de las investigaciones, que un mismo hecho traumático puede ser recordado de una manera vívida, consistente, o, por el contrario, fragmentado, o incluso no recordarse. Un mejor conocimiento de los factores de influencia quizá podría explicar las diferencias entre memorias traumáticas y memorias autobiográficas sobre hechos neutros.

\section{Variables de influencia sobre las carac- terísticas de las memorias traumáticas}

El primer factor que destacamos es el estrés. Así lo indican todos aquellos estudios que advierten de su influencia en la memoria ${ }^{30,31}$. Su relación es compleja, ya que, como hemos visto, las experiencias estresantes traumáticas podrían producir la sensación de recuerdos intensos, vívidos y persistentes, al tiempo que produce un deterioro significativo de la capacidad de atención y memoria. La vivencia del estrés no está en función de las características del trauma si no de la manera en que cada organismo percibe y reacciona al estímulo traumático. Como cada organismo percibe y reacciona al estrés está en función de diferentes factores, entre los que ocupan 
un lugar importante el temperamento y el apego ${ }^{32}$. Derivado de la combinación de ambos factores contaremos con un tipo de capacidad u otra para desplegar las estrategias de afrontamiento necesarias para que el organismo responda al estímulo traumático sin perturbar seriamente su equilibrio y, por tanto, minimizando su efecto sobre la memoria o la atención. En el caso de una agresión, en un entorno donde se produce una reacción sensible, reguladora y protectora, probablemente se minimizarán los efectos traumáticos.

En cualquier caso, la intensidad de la emoción asociada al hecho y el grado de implicación parecen ser factores importantes. Por regla general, los hechos autobiográficos con una implicación emocional importante se recuerdan más detalladamente que los hechos rutinarios con baja implicación emocional ${ }^{33}$, lo que no implica que todos los detalles recordados sean exactos ni la memoria generada sea inmune al paso del tiempo. En un estudio llevado a cabo con población Palestina de la franja de Gaza ${ }^{34}$ los resultados encontrados mostraron que ser protagonista del hecho traumático lleva a memorias más fragmentadas, más confusas, con sentimientos asociados más intensos, y memorias más accesibles, dado que tienden más a re-experimentar y a pensar en los hechos vividos, en comparación con los recuerdos de los sujetos que fueron testigos del suceso y cuyas emociones asociadas fueron menos intensas. En la misma dirección, se ha evaluado el efecto de la implicación en el hecho traumático sobre la exactitud de los recuerdos ${ }^{35}$, encontrándose que el grado, tipo (central vs periférico) y exactitud de los detalles recordados por los sujetos estaba relacionado directamente con la implicación del sujeto.

\section{Exactitud e inmutabilidad de las memorias traumáticas: "jamás se me olvidará"}

Es muy probable que ciertos detalles de nuestras memorias autobiográficas sobre hechos traumáticos que damos por exactos hayan sido "creados" posteriormente ${ }^{36,37}$. Ost y colaboradores ${ }^{38}$ encontraron, en un experimento sobre memorias de hechos traumáticos, que un $40 \%$ de sujetos creían haber visto escenas falsas sobre los atentados de Londres de 2005, que habían sido generadas por los investigadores. En la misma dirección, se han realizado numerosos estudios sobre los recuerdos acerca de los atentados terroristas del 11-S en Nueva York ${ }^{39-44}$, mostrando interesantes resultados que en esencia confirman la alteración de este tipo de memorias con el paso del tiempo. Así, por ejemplo, Schmidt ${ }^{45}$ encontró que los hechos centrales se recuerdan con más consistencia que los periféricos, pero los recuerdos sobre este suceso contenían abundantes errores procedentes de una inapropiada reconstrucción de los hechos. Los sujetos más afectados emocionalmente mostraron un peor recuerdo y más inconsistencias respecto a los detalles periféricos que los sujetos menos afectados. Toda aquella información que procede de la estimación del sujeto y no de su percepción directa será más susceptible de modificarse a lo largo del tiempo.

Sin embargo, es corriente escuchar a víctimas de sucesos violentos decir, por ejemplo, "me ha impresionado tanto, que nunca lo olvidaré" porque la mayoría de la gente piensa que cuanto más violento sea un suceso más impactará y, por tanto, mejor será después su recuerdo. La realidad es que se ha probado cómo los sucesos que implican un mayor grado de violencia se recuerdan peor ${ }^{46}$. Clifford y Scott explican este efecto indicando que el sujeto experimenta mayor estrés cuanta mayor violencia implica el suceso, y el estrés afecta negativamente a los procesos cognitivos como la atención, la percepción y la memoria. La falta de recursos atencionales que genera el estrés dificulta el procesamiento en profundidad de la información, así los sujetos pueden procesar la información más básica de forma pre-atencional, pero no integrar después adecuadamente toda esa información en una representación completa y exacta, generando conjunciones ilusorias, de modo que el sujeto puede tener todas las piezas del puzzle, pero montarlo de forma errónea, dando lugar a un relato de los hechos diferente a lo acontecido en realidad ${ }^{47}$.

\section{Accesibilidad de lo recuerdos sobre hechos traumáticos}

En algunas ocasiones se ha afirmado que las memorias sobre hechos traumáticos podrían quedar "reprimidas" o dar lugar a fenómenos disociativos que generen una incapacidad para recordar los hechos ${ }^{48}$. Este tipo de amnesias psicógenas se han relacionado con el trastorno de estrés post-traumático. Según estas hipótesis, el recuerdo no se perdería, sino que permanecería en la memoria aunque inaccesible. De este modo, mucho tiempo después, un acontecimiento similar o en cualquier caso los indicios de recuperación adecuados podrían hacerlo consciente de nuevo. A estos recuerdos antes reprimidos y ahora accesibles se les ha denominado 
como memorias recuperadas. No obstante, los estudios experimentales al respecto han fracasado al tratar de probar la existencia de este tipo de fenómenos. Más bien al contrario, algunos autores ${ }^{49-51}$ afirman que las memorias traumáticas se recuerdan más y mejor que las memorias neutras. En un estudio sobre recuerdos acerca de agresiones sexuales, Porter y Birt $^{52}$ encuentran que tienden a recordarse con mayor frecuencia que otras memorias autobiográficas, y en los pocos casos en los que encuentran que este tipo de sucesos se han olvidado ( $4,6 \%$ del total) se debe más a un intento deliberado de no recordar que a una memoria reprimida o disociada. La clave que explique esta controversia probablemente podría estar en que la existencia de las amnesias psicógenas se sustenta fundamentalmente en las manifestaciones de víctimas y en población clínica, sin un estudio acerca de la verosimilitud de dichas manifestaciones y donde en ocasiones se confunde la incapacidad o dificultad para hablar del suceso con el olvido. Del mismo modo, no deberíamos confundir la experiencia fenomenológica que genera el recuerdo con el recuerdo en sí mismo. En esta dirección Geraerts y colaboradores ${ }^{53}$ a partir de una serie de investigaciones afirman que los sujetos podrían tener la impresión subjetiva de haber reprimido las memorias sobre hechos traumáticos, aun cuando podría ser que hayan olvidado que las habían recordado previamente.

En cualquier caso, no parece que el recuerdo de hechos traumáticos sea inmune al deterioro y la distorsión por efecto de la sugerencia de información falsa $^{54}$. Independientemente de la controversia en torno a la existencia o no de las memorias recuperadas, conviene señalar que una cosa es no querer recordar y otra muy diferente olvidar realmente. Aún cuando muchas de las víctimas de un suceso traumático tratan de no recordar, lo cierto es que la accesibilidad de este tipo de memorias no parece verse comprometida, aunque a estas personas les resulte difícil hablar de lo ocurrido. Esto puede explicar por qué un hecho sucedido en la infancia, que durante mucho tiempo permaneció inaccesible, ante nuevas claves de recuperación años después pueda dar lugar a su recuerdo espontáneo, aunque ahora lo que se recupera esté sesgado por los conocimientos y experiencias posteriores, que pueden dar lugar a una interpretación del hecho diferente de la original.

\section{Conclusiones}

En resumen, el debate en torno a las memorias traumáticas nos lleva a afirmar que no todos recordamos de la misma manera los hechos traumáticos. Ante un mismo evento traumático un porcentaje de los implicados tendrán un recuerdo de lo sucedido de manera muy vívida a lo largo de los años, otro porcentaje lo recordará de manera fragmentada, otro tanto no tendrá diferencias en su manera de recordar con respecto a otros eventos autobiográficos, y, por último, algunos podrían tener problemas a la hora de acceder a algunos detalles de lo que ocurrió.

De la misma manera que encontramos diferencias a la hora de recordar, también encontraremos diferencias en los efectos psicológicos, emocionales y psiquiátricos que puede conllevar la experiencia traumática. Así, habrá víctimas de hechos traumáticos que tengan una enorme capacidad de resiliencia y consigan superar el trauma sin demasiado esfuerzo, y habrá personas que lleguen a padecer un TEPT que puede durar años o incluso un trastorno de personalidad severo. La investigación y la práctica clínica nos ha evidenciado que hay factores que van a determinar el impacto psicológico que el trauma conlleve en la víctima de un hecho traumático.

Sorprende que a diferencia del contexto clínico y experimental que investiga las diferencias individuales del impacto del trauma, que nadie niega, así como los factores que condicionan dicho impacto, en el estudio de las memorias traumáticas no se abogue más por estudiar los factores que median en el recuerdo intersubjetivo e intrasubjetivo de una experiencia traumática. Así, todavía serán necesarias más investigaciones para poder establecer más específicamente las diferencias entre los recuerdos de hechos traumáticos y otro tipo de sucesos, y evaluar los factores que podrían condicionarlos (gravedad del hecho, tiempo transcurrido, duración del suceso, implicación, etc.).

Por último, y no por ello menos importante, debemos considerar, cuando nos movemos en contextos forenses, los trabajos en torno a la exactitud de las memorias traumáticas, que advierten de que no hay ningún tipo de recuerdo, independientemente del tipo de huella que haya dejado, que no sea susceptible de distorsión. La memoria almacena interpretaciones de la realidad, no la realidad misma, y trata de recuperar una información de un hecho de la infancia en un contexto adulto, significa reinterpretar la información entonces almacenada. Que no lo recordemos no quiere decir que no sucediera, pero que lo recordemos tampoco implica que ocurriera. 


\section{Bibliografía}

1. Nemeroff CB, Bremner JD, Foa EB, Mayberg HS, North CS Stein MB. Posttraumatic stress disorder: A state-of-science review. J Psychiatric Research 2006;40:1-21.

2. Silver RC, Holman EA, Mclntosh. Nationwide longitudinal study for psychological responses to September 11. JAMA 2002;288:1235-44.

3. Manzanero AL. Recuerdo de hechos traumáticos: de la introspección al estudio objetivo. Rev Psicopat Clin, Leg Forense 2010;10:1-22.

4. Brewin CR. Autobiographical memory for trauma: Update on four controversies. Memory 2007; 15:227-48.

5. Brown R, Kulik J. Flashbulb memories. Cognition 1977;5:73-99.

6. Neisser U, Harsch N. Phantom flashbulbs: false recollections of hearing the news about Challenger. In Winograd E, Neisser U, eds. Affect and accuracy: Studies of 'flashbulb' memories. New York: Cambridge University Press; 1992. p. 9-31.

7. Peace KA, Porter S. A longitudinal investigation of the reliability of memories for trauma and other emotional experiences. App Cog Psy. 2004;18:1143-59.

8. Peace KA, Porter S, Brinke L. Are memories for sexually traumatic events "special"? A within-subjects investigation of trauma and memory in a clinical sample. Memory 2008; 16: 10-21.

9. Pillemer DB. Flashbulb memories of the assassination attempt on President Reagan. Cognition 1984;16:63-80

10. Peace KA, Porter S. A longitudinal investigation of the reliability of memories for trauma and other emotional experiences. App Cog Psy 2004;18:1143-59.

11. Peace KA, Porter S, Brinke L. Are memories for sexually traumatic events "special"? A within-subjects investigation of trauma and memory in a clinical sample. Memory 2008;16:10-21.

12. Christianson SA. Emotional stress and eyewitness memory: A critical review. Psy Bull 1992;112:284309.

13. Loftus EF, Loftus GR, Messo J. Some facts about weapon focus. Law Hum Beh 1987;11:55-62.

14. Byrne CA, Hyman IE, Scott KL. Comparisons of memories for traumatic events and other experiences. App Cog Psychology 2001;15:119-33.

15. Kihlstrom JF. The trauma-memory argument and recovered memory therapy. In Pezdek K, Banks WP, eds. The recovered memory/false memory debate. San Diego, CA: Academic Press; 1996. p. 297-311.
16. Neisser U, Harsch N. Phantom flashbulbs: false recollections of hearing the news about Challenger. In Winograd E, Neisser U, eds. Affect and accuracy: Studies of 'flashbulb' memories. New York: Cambridge University Press; 1992. p. 9-31.

17. Southwick SM, Morgan CA, Nicolaou AL, Charney DS. Consistency of memory for combat-related traumatic events in veterans of Operation Desert Storm. Am J Psychiatry 1997;154:173-7.

18. Yuille JC, Cutshall JL. A case study of eyewitness memory of a crime. J App Psy 1986; 71:291-301.

19. Van der Kolk BA. Trauma and memory. In Van der Kolk BA, McFarlane NC, Wesaeth L, eds. Traumatic Stress. Nueva York: Guilford; 1996. p. 279-302.

20. Van der Kolk BA. The psychobiology of posttraumatic stress dissorder. J Clin Psych 1997;58:16-24.

21. Herman JL. Trauma and recovery. New York: Basic Books; 1992.

22. Yuille JC, Cutshall JL. A case study of eyewitness memory of a crime. J App Psy 1986; 71:291-301

23. Baddeley A. Memoria autobiográfica. In A Baddeley, MW Eysenck y MC Anderson. Memoria. Madrid: Alianza Editorial; 2010. p. 165-91.

24. Nemeroff C, Bremner J, Foa E, Mayberg H, North C, Stein, M. Posttraumatic stress disorder: a stateof-science review. J Psychiatric Research, 2006;40:1-21.

25. Cottecin O, Vaiva G, Huron C, Devos P, Ducroca F, Jouvent R, Goudemand M, Thomas P. Directed forgetting in PTSD: a comparative study versus normal controls. Jurnal of Psiquiatric Research 2006;40:70-80.

26. Loftus EF. The reality of repressed memories. Ame Psy 1993; 48: 518-37.

27. Manzanero AL, López B. Características de los recuerdos autobiográficos sobre sucesos traumáticos. Bol Psicología 2007;90:7-17.

28. López B, Manzanero AL, El-Astal S, Aróztegui J (en revisión). Phenomenological features of autobiographical memories for negative events.

29. Manzanero AL, Contreras MJ, López B, El-Astal S (en revisión). Implication degree and emotion in autobiographical memories for negative events.

30. Kim J, Diamond D. The stresse hippocampus, synaptic plasticity and lost memories. Nouroscience 2002, vol.3;453-62.

31. McEwen, B.S. (2000) Effects of adverse experiences for brain estructure and function. Biol Psychiatry 48,721-31. 
32. Nemeroff C, Bremner J, Foa E, Mayberg H, North C, Stein, M. Posttraumatic stress disorder: a state of science review. Journal os Psiquiatric Research, 2006, vol. 40 1-21.

33. Talarico JM, LaBar KS, Rubin DC. Emotional intensity predicts autobiographical memory experience. Mem Cog 2004;32:1118-32.

34. Manzanero AL, Contreras MJ, López B, El-Astal S (en revisión). Implication degree and emotion in autobiographical memories for negative events.

35. Nachson I, Slavutskay-Tsukerman I. Effect of personal involvement in traumatic events on memory: The case of the Dolphinarium explosion. Memory 2010;18:241-51.

36. Brown R, Kulik J. Flashbulb memories. Cognition 1977; 5: 73-99.

37. Pillemer DB. Flashbulb memories of the assassination attempt on President Reagan. Cognition 1984;16:63-80.

38. Ost J, Granhag PA, Udell J, Hjelmsäter ER. Familiarity breeds distortion: The effects of media exposure on false reports concerning media coverage of the terrorist attacks in London on 7 July 2005. Memory 2007; 16:76-85.

39. Lee PJ, Brown NR. Delay related changes in personal memories for September 11, 2001. App Cog Psy 2003;17:1007-15.

40. Luminet O, Curci A, Marsh EJ, Wessel I, Constantin C, Gencoz F, Yoko M. The cognitive, emotional, and social impacts of the September 11 attacks: Group differences in memory for the reception context and the determinants of flashbulb memory. J Gral Psy 2004;13:197-224.

41. Pezdek K. Event memory and autobiographical memory for events of September 11, 2001. App Cog Psy 2003; 17: 1033-45.

42. Schmidt SR. Autobiographical memories for the September 11 th attacks: Reconstructive errors and emotional impairment of memory. Mem Cog 2004;32:443-54.
43. Talarico JM, Rubin DC. Flashbulb memories are special after all; in phenomenology, not accuracy. App Cog Psy 2007;21:557-78.

44. Tekcam AI, Ece B, Gülgöz S, Er N. Autobiographical and event memory for 9/11: Changes across one year. App Cog Psy 2003;17:1057-66.

45. Schmidt SR. Autobiographical memories for the September 11th attacks: Reconstructive errors and emotional impairment of memory. Mem Cog 2004;32:443-54.

46. Clifford BR, Scott J. Individual and situational factors in eyewitness testimony. J App Psy 1978;63:352-9.

47. Manzanero AL. Memoria de testigos: Obtención y valoración de la prueba testifical. Madrid: Pirámide, 2010.

48. Van der Kolk BA, Fisler R. Dissociation and the fragmentary nature of traumatic memories: Overview and exploratory study. J Traumatic Stress 1995;8:505-25.

49. Terr LC. Chowchilla revisited: The effects of psychic trauma four years after a school-bus kidnapping. American J Psy 1983;140:1543-50.

50. Wagenaar WA, Groeneweg J. The memory of concentration camp survivors. App Cog Psy 1990;4:77-87.

51. Yuille JC, Cutshall JL. A case study of eyewitness memory of a crime. J App Psy 1986;71:291-301.

52. Porter S, Birt AR. Is traumatic memory special? A comparison of traumatic memory characteristics with memory for other emotional life experiences. App Cog Psy 2001; 15: 101-17.

53. Geraerts E, McNally RJ, Jelicic M, Merckelbach H, Raymaekers L. Linking thought suppression and recovered memories of childhood sexual abuse. Memory $2007 ; 16: 22-8$.

54. Paz-Alonso PM, Goodman GS. Trauma and memory: Effects of post-event misinformation, retrieval order, and retention interval. Memory 2007; 16:58-75. 